\title{
Inequalities for Katugampola conformable partial derivatives
}

\author{
Chang-Jian Zhao ${ }^{1 *}$ and Wing-Sum Cheung ${ }^{2}$
}

\section{"Correspondence:} chjzhao@163.com

${ }^{1}$ Department of Mathematics, China Jiliang University, Hangzhou, P.R. China

Full list of author information is available at the end of the article
The Author(s) 2019. This article is distributed under the terms of the Creative Commons Attribution 4.0 International License (http://creativecommons.org/licenses/by/4.0/), which permits unrestricted use, distribution, and reproduction in any medium, provided you give appropriate credit to the original author(s) and the source, provide a link to the Creative Commons license, and indicate if changes were made.

\begin{abstract}
In the paper, we introduce two concepts of Katugampola conformable partial derivatives and $\alpha$-conformable integrals. As applications, we establish Opial type inequalities for Katugampola conformable partial derivatives and $\alpha$-conformable integrals. The new inequalities in special cases yield some of the recent results on inequality of this type.
\end{abstract}

MSC: 26D15; 26A51

Keywords: Convex function; Conformable fractional integrals; Katugampola conformable derivative; Opial's inequality; Jensen's inequality

\section{Introduction}

In 1960, Opial [1] established the following interesting and important inequality.

Theorem A Suppose that $f \in C^{1}[0, a]$ satisfies $f(0)=f(a)=0$ and $f(x)>0$ for all $x \in(0, a)$. Then the inequality holds

$$
\int_{0}^{a}\left|f(x) f^{\prime}(x)\right| d x \leq \frac{a}{4} \int_{0}^{a}\left(f^{\prime}(x)\right)^{2} d x
$$

where this constant a/4 is best possible.

Opial's inequality and its generalizations, extensions, and discretizations play a fundamental role in establishing the existence and uniqueness of initial and boundary value problems for ordinary and partial differential equations as well as difference equations [2-6]. Inequality (1.1) has received considerable attention, and a large number of papers dealing with new proofs, extensions, generalizations, variants, and discrete analogues of Opial's inequality have appeared in the literature [7-18].

Recently, some new Opial's inequalities for the conformable fractional integrals have been established (see [19-22]). In the paper, we introduce two new concepts of Katugampola conformable partial derivatives and $\alpha$-conformable integrals. As applications, we establish some Opial type inequalities for Katugampola conformable partial derivatives and $\alpha$-conformable integrals. 


\section{Inequalities for Katugampola conformable partial derivatives}

We recall the well-known Katugampola derivative formulation of conformable derivative of order for $\alpha \in(0,1]$ and $t \in[0, \infty)$, given by

$$
D_{\alpha}(f)(t)=\lim _{\varepsilon \rightarrow 0} \frac{f\left(t e^{\varepsilon t^{-\alpha}}\right)-f(t)}{\varepsilon},
$$

and

$$
D_{\alpha}(f)(0)=\lim _{t \rightarrow 0} D_{\alpha}(f)(t)
$$

provided the limits exist. If $f$ is fully differentiable at $t$, then

$$
D_{\alpha}(f)(t)=t^{1-\alpha} \frac{d f}{d t}(t)
$$

A function $f$ is $\alpha$-differentiable at a point $t \geq 0$ if the limits in (2.1) and (2.2) exist and are finite. Inspired by this, we propose a new concept of $\alpha$-conformable partial derivative. In the way of (2.1), we define $\alpha$-conformable partial derivative.

Definition 2.1 ( $\alpha$-conformable partial derivative) Let $\alpha \in(0,1]$ and $s, t \in[0, \infty)$. Suppose that $f(s, t)$ is a continuous function and partially derivable, the $\alpha$-conformable partial derivative at a point $s \geq 0$, denoted by $\frac{\partial}{\partial s}(f)_{\alpha}(s, t)$, is defined by

$$
\frac{\partial}{\partial s}(f)_{\alpha}(s, t)=\lim _{\varepsilon \rightarrow 0} \frac{f\left(s e^{\varepsilon s^{-\alpha}}, t\right)-f(s, t)}{\varepsilon},
$$

provided the limits exist, and is called $\alpha$-conformable partially derivable.

To generalize Definition 2.1, we give the following definition.

Definition 2.2 (Katugampola conformable partial derivative) Let $\alpha \in(0,1]$ and $s, t \in$ $[0, \infty)$. Suppose that $f(s, t)$ and $\frac{\partial}{\partial s}(f)_{\alpha}(s, t)$ are continuous functions and partially derivable, the Katugampola conformable partial derivative, denoted by $\frac{\partial^{2}}{\partial s \partial t}(f)_{\alpha^{2}}(s, t)$, is defined by

$$
\frac{\partial^{2}}{\partial s \partial t}(f)_{\alpha^{2}}(s, t)=\lim _{\varepsilon \rightarrow 0} \frac{\frac{\partial}{\partial s}(f)_{\alpha}\left(s, t e^{\varepsilon t^{-\alpha}}\right)-\frac{\partial}{\partial s}(f)_{\alpha}(s, t)}{\varepsilon},
$$

provided the limits exist, and is called Katugampola conformable partially derivable.

Definition 2.3 ( $\alpha$-conformable integral) Let $\alpha \in(0,1], 0 \leq a<b$, and $0 \leq c<d$. A function $f(x, y):[a, b] \times[c, d] \rightarrow \mathbb{R}$ is $\alpha$-conformable integrable if the integral

$$
\int_{a}^{b} \int_{c}^{d} f(x, y) d_{\alpha} x d_{\alpha} y:=\int_{a}^{b} \int_{c}^{d}(x y)^{\alpha-1} f(x, y) d x d y
$$

exists and is finite. 
Lemma 2.1 Let $\alpha \in(0,1], s, t \in[0, \infty)$, and $f(s, t), g(s, t)$ be Katugampola conformable partially differentiable, then

$$
\frac{\partial^{2}}{\partial s \partial t}(f \circ g)_{\alpha^{2}}(s, t)=f^{\prime}(g(s, t)) \cdot \frac{\partial^{2}}{\partial s \partial t}(g)_{\alpha^{2}}(s, t)+\frac{\partial}{\partial t}(g)_{\alpha}(s, t) \cdot \frac{\partial}{\partial t}\left(f^{\prime}(g(s, t))\right)_{\alpha}(s, t),
$$

where $f$ has derivative at $g(s, t)$.

Proof From Definitions 2.1 and 2.2, we obtain

$$
\begin{aligned}
\frac{\partial}{\partial s}(f \circ g)_{\alpha}(s, t) & =\frac{\partial}{\partial s}(f(g(s, t)))_{\alpha}(s, t) \\
& =s^{1-\alpha} \frac{\partial}{\partial s}(f(g(s, t))) \\
& =s^{1-\alpha} f^{\prime}(g(s, t)) \frac{\partial}{\partial s}(g(s, t)) \\
& =f^{\prime}(g(s, t)) \frac{\partial}{\partial s}(g)_{\alpha}(s, t) .
\end{aligned}
$$

Hence

$$
\begin{aligned}
\frac{\partial^{2}}{\partial s \partial t}(f \circ g)_{\alpha^{2}}(s, t) & =\frac{\partial}{\partial t}\left(\frac{\partial}{\partial s}(f \circ g)_{\alpha}(s, t)\right)_{\alpha}(s, t) \\
& =\frac{\partial}{\partial t}\left(f^{\prime}(g(s, t)) \frac{\partial}{\partial s}(g)_{\alpha}(s, t)\right)_{\alpha}(s, t) \\
& =t^{1-\alpha} \frac{\partial}{\partial t}\left(f^{\prime}(g(s, t)) \cdot \frac{\partial}{\partial s}(g)_{\alpha}(s, t)\right) \\
& =t^{1-\alpha} \frac{\partial}{\partial t}\left(f^{\prime}(g(s, t))\right) \cdot \frac{\partial}{\partial t}(g)_{\alpha}(s, t)+t^{1-\alpha} f^{\prime}(g(s, t)) \cdot \frac{\partial}{\partial t}\left(\frac{\partial}{\partial s}(g)_{\alpha}(s, t)\right) \\
& =\frac{\partial}{\partial t}(g)_{\alpha}(s, t) \cdot \frac{\partial}{\partial t}\left(f^{\prime}(g(s, t))\right)_{\alpha}(s, t)+f^{\prime}(g(s, t)) \cdot \frac{\partial^{2}}{\partial s \partial t}(g)_{\alpha^{2}}(s, t) .
\end{aligned}
$$

This completes the proof.

This similar chain rule theorem is important, but it is also understood. In order for the reader to better understand this theorem, we give another proof below.

Second proof Let

$$
\delta=g\left(s e^{\varepsilon s^{-\alpha}}, t\right)-g(s, t)
$$

Obviously, if $\varepsilon \rightarrow 0$, then $\delta \rightarrow 0$. From the hypotheses, we obtain

$$
\begin{aligned}
\frac{\partial^{2}}{\partial s \partial t}(f \circ g)_{\alpha^{2}}(s, t) & =\frac{\partial}{\partial t}\left(\frac{\partial}{\partial s}(f(g(s, t)))_{\alpha}(s, t)\right)_{\alpha}(s, t) \\
& =\frac{\partial}{\partial t}\left(\lim _{\varepsilon \rightarrow 0} \frac{f\left(g\left(s e^{\varepsilon s^{-\alpha}}, t\right)\right)-f(g(s, t))}{\varepsilon}\right)_{\alpha}(s, t) \\
& =\frac{\partial}{\partial t}\left(\lim _{\delta \rightarrow 0} \frac{f(g(s, t)+\delta)-f(g(s, t))}{\delta} \cdot \lim _{\varepsilon \rightarrow 0} \frac{\delta}{\varepsilon}\right)_{\alpha}(s, t)
\end{aligned}
$$




$$
\begin{aligned}
& =\frac{\partial}{\partial t}\left(f^{\prime}(g(s, t)) \frac{\partial}{\partial s}(g)_{\alpha}(s, t)\right)_{\alpha}(s, t) \\
& =f^{\prime}(g(s, t)) \cdot \frac{\partial^{2}}{\partial s \partial t}(g)_{\alpha^{2}}(s, t)+\frac{\partial}{\partial t}(g)_{\alpha}(s, t) \cdot \frac{\partial}{\partial t}\left(f^{\prime}(g(s, t))\right)_{\alpha}(s, t) .
\end{aligned}
$$

This completes the proof.

Theorem 2.1 Let $p(s, t), u(s, t):[a, b] \times[c, d] \rightarrow \mathbb{R}$ with $a, c \geq 0$ be Katugampola conformable partially derivable such that $\frac{\partial^{2}}{\partial s \partial t}(p)_{\alpha^{2}}(s, t)>0, \alpha \in(0,1]$ and $p(a, c)=p(a, d)=$ $p(b, c)=p(b, d)=0$, and $F$ be derivable on $[0, \infty)$ and $F^{\prime}$ be increasing. Let $\varphi$ be a convex and increasing function on $[0, \infty)$, and define

$$
z(s, t)=\int_{a}^{s} \int_{c}^{t} \frac{\partial^{2}}{\partial \sigma \partial \tau}(p)_{\alpha^{2}}(\sigma, \tau) \cdot \varphi\left(\frac{\left|\frac{\partial^{2}}{\partial \sigma \partial \tau}(u)_{\alpha^{2}}(\sigma, \tau)\right|}{\frac{\partial^{2}}{\partial \sigma \partial \tau}(p)_{\alpha^{2}}(\sigma, \tau)}\right) d_{\alpha} \sigma d_{\alpha} \tau .
$$

Then

$$
\begin{aligned}
\int_{a}^{b} \int_{c}^{d}\left\{\frac{\partial^{2}}{\partial s \partial t}(z)_{\alpha^{2}}(s, t) \cdot F^{\prime}\left(p(s, t) \cdot \varphi\left(\frac{|u(s, t)|}{p(s, t)}\right)\right)\right. \\
\left.+\frac{\partial}{\partial t}(z)_{\alpha}(s, t) \cdot \frac{\partial}{\partial t}\left(F^{\prime}(z(s, t))\right)_{\alpha}(s, t)\right\} d_{\alpha} s d_{\alpha} t \\
\leq F\left(\int_{a}^{b} \int_{c}^{d} \frac{\partial^{2}}{\partial s \partial t}(p)_{\alpha^{2}}(s, t) \cdot \varphi\left(\frac{\left|\frac{\partial^{2}}{\partial s \partial t}(u)_{\alpha^{2}}(s, t)\right|}{\frac{\partial^{2}}{\partial s \partial t}(p)_{\alpha^{2}}(s, t)}\right) d_{\alpha} s d_{\alpha} t\right),
\end{aligned}
$$

where

$$
\frac{\partial}{\partial t}\left(F^{\prime}(z(s, t))\right)_{\alpha}(s, t)=t^{1-\alpha} \frac{\partial}{\partial t} F^{\prime}(z(s, t)) .
$$

Proof Let

$$
y(s, t)=\int_{a}^{s} \int_{c}^{t}\left|\frac{\partial^{2}}{\partial s \partial t}(u)_{\alpha^{2}}(\sigma, \tau)\right| d_{\alpha} \sigma d_{\alpha} \tau
$$

such that

$$
\frac{\partial^{2}}{\partial s \partial t}(y)_{\alpha^{2}}(s, t)=\left|\frac{\partial^{2}}{\partial s \partial t}(u)_{\alpha^{2}}(s, t)\right|
$$

and $y(s, t) \geq|u(s, t)|$. Since $\varphi$ is convex and increasing, by using Jensen's inequality, we get

$$
\begin{aligned}
\varphi\left(\frac{|u(s, t)|}{p(s, t)}\right) & \leq \varphi\left(\frac{y(s, t)}{p(s, t)}\right) \\
& =\varphi\left(\frac{\int_{a}^{s} \int_{c}^{t} \frac{\partial^{2}}{\partial \sigma \partial \tau}(p)_{\alpha^{2}}(\sigma, \tau) \frac{\left|\frac{\partial^{2}}{\partial \sigma \partial \tau}(u)_{\alpha^{2}}(\sigma, \tau)\right|}{\frac{\partial^{2}}{\partial \sigma \tau \tau}(p)_{\alpha^{2}}(\sigma, \tau)} d_{\alpha} \sigma d_{\alpha} \tau}{\int_{a}^{s} \int_{c}^{t} \frac{\partial^{2}}{\partial \sigma \partial \tau}(p)_{\alpha^{2}}(\sigma, \tau) d_{\alpha} \sigma d_{\alpha} \tau}\right)
\end{aligned}
$$




$$
\begin{aligned}
& \leq \frac{1}{p(s, t)} \int_{a}^{s} \int_{c}^{t} \frac{\partial^{2}}{\partial \sigma \partial \tau}(p)_{\alpha^{2}}(\sigma, \tau) \cdot \varphi\left(\frac{\left|\frac{\partial^{2}}{\partial \sigma \partial \tau}(u)_{\alpha^{2}}(\sigma, \tau)\right|}{\frac{\partial^{2}}{\partial \sigma \partial \tau}(p)_{\alpha^{2}}(\sigma, \tau)}\right) d_{\alpha} \sigma d_{\alpha} \tau \\
& =\frac{1}{p(s, t)} \int_{a}^{s} \int_{c}^{t} \frac{\partial^{2}}{\partial \sigma \partial \tau}(p)_{\alpha^{2}}(\sigma, \tau) \cdot \varphi\left(\frac{\frac{\partial^{2}}{\partial \sigma \partial \tau}(y)_{\alpha^{2}}(\sigma, \tau)}{\frac{\partial^{2}}{\partial \sigma \partial \tau}(p)_{\alpha^{2}}(\sigma, \tau)}\right) d_{\alpha} \sigma d_{\alpha} \tau .
\end{aligned}
$$

From (2.9) and noting that $F^{\prime}$ is increasing, and Lemma 2.1, (2.7) and in view of that $F$ is derivable on $[0, \infty)$, we obtain

$$
\begin{aligned}
& \int_{a}^{b} \int_{c}^{d}\left\{\frac{\partial^{2}}{\partial s \partial t}(z)_{\alpha^{2}}(s, t) \cdot F^{\prime}\left(p(s, t) \cdot \varphi\left(\frac{|u(s, t)|}{p(s, t)}\right)\right)\right. \\
& \left.+\frac{\partial}{\partial t}(z)_{\alpha}(s, t) \cdot \frac{\partial}{\partial t}\left(F^{\prime}(z(s, t))\right)_{\alpha}(s, t)\right\} d_{\alpha} s d_{\alpha} t \\
& \leq \int_{a}^{b} \int_{c}^{d}\left\{\frac{\partial^{2}}{\partial s \partial t}(z)_{\alpha^{2}}(s, t) \cdot F^{\prime}(z(s, t))\right. \\
& \left.+\frac{\partial}{\partial t}(z)_{\alpha}(s, t) \cdot \frac{\partial}{\partial t}\left(F^{\prime}(z(s, t))\right)_{\alpha}(s, t)\right\} d_{\alpha} s d_{\alpha} t \\
& =\int_{a}^{b} \int_{c}^{d} \frac{\partial^{2}}{\partial s \partial t}(F \circ z)_{\alpha^{2}}(s, t) d_{\alpha} s d_{\alpha} t \\
& =\int_{a}^{b} \int_{c}^{d} \frac{\partial^{2}}{\partial s \partial t}\left(F \left(\int_{a}^{s} \int_{b}^{t} \frac{\partial^{2}}{\partial \sigma \partial \tau}(p)_{\alpha^{2}}(\sigma, \tau)\right.\right. \\
& \left.\left.\cdot \varphi\left(\frac{\frac{\partial^{2}}{\partial \sigma \partial \tau}(y)_{\alpha^{2}}(\sigma, \tau)}{\frac{\partial^{2}}{\partial \sigma \partial \tau}(p)_{\alpha^{2}}(\sigma, \tau)}\right) d_{\alpha} \sigma d_{\alpha} \tau\right)\right)_{\alpha^{2}}(s, t) d_{\alpha} s d_{\alpha} t \\
& =F\left(\int_{a}^{b} \int_{c}^{d} \frac{\partial^{2}}{\partial \sigma \partial \tau}(p)_{\alpha^{2}}(\sigma, \tau) \cdot \varphi\left(\frac{\frac{\partial^{2}}{\partial \sigma \partial \tau}(y)_{\alpha^{2}}(\sigma, \tau)}{\frac{\partial^{2}}{\partial \sigma \partial \tau}(p)_{\alpha^{2}}(\sigma, \tau)}\right) d_{\alpha} \sigma d_{\alpha} \tau\right) \\
& =F\left(\int_{a}^{b} \int_{c}^{d} \frac{\partial^{2}}{\partial s \partial t}(p)_{\alpha^{2}}(s, t) \cdot \varphi\left(\frac{\left|\frac{\partial^{2}}{\partial s \partial t}(u)_{\alpha^{2}}(s, t)\right|}{\frac{\partial^{2}}{\partial s \partial t}(p)_{\alpha^{2}}(s, t)}\right) d_{\alpha} s d_{\alpha} t\right) .
\end{aligned}
$$

This completes the proof.

Remark 2.1 Putting $\varphi(x)=x$ in (2.7), we have

$$
\begin{aligned}
\int_{a}^{b} \int_{c}^{d} & \left\{\left|\frac{\partial^{2}}{\partial s \partial t}(u)_{\alpha^{2}}(s, t)\right| \cdot F^{\prime}(|u(s, t)|)\right. \\
& \left.+\frac{\partial}{\partial t}(y)_{\alpha}(s, t) \cdot \frac{\partial}{\partial t}\left(F^{\prime}(y(s, t))\right)_{\alpha}(s, t)\right\} d_{\alpha} s d_{\alpha} t \\
\leq & F\left(\int_{a}^{b} \int_{c}^{d}\left|\frac{\partial^{2}}{\partial s \partial t}(u)_{\alpha^{2}}(s, t)\right| d_{\alpha} s d_{\alpha} t\right)
\end{aligned}
$$

where

$$
y(s, t)=\int_{a}^{s} \int_{c}^{t}\left|\frac{\partial^{2}}{\partial s \partial t}(u)_{\alpha^{2}}(\sigma, \tau)\right| d_{\alpha} \sigma d_{\alpha} \tau .
$$


This inequality (2.10) is just a two-dimensional generalization of the following inequality which was established in [20] and [21]:

$$
\int_{a}^{b}\left|D_{\alpha} u(t)\right| \cdot F^{\prime}(|u(t)|) d_{\alpha} t \leq F\left(\int_{a}^{b}\left|D_{\alpha} u(t)\right| d_{\alpha} t\right) .
$$

Theorem 2.2 Let $\alpha, p(s, t), u(s, t), z(s, t), \varphi, F$ be as in Theorem 2.1 and replace $[a, b] \times[c, d]$ by $[0, a] \times[0, b]$. Let $h$ be a concave and increasing function on $[0, \infty)$, and $\phi$ be a continuous and positive function on $[0, \infty)$ and such that

$$
\frac{\partial^{2}}{\partial s \partial t}(F \circ z)_{\alpha^{2}}(s, t) \cdot \phi\left(\frac{1}{\frac{\partial^{2}}{\partial s \partial t}(z)_{\alpha^{2}}(s, t)}\right) \leq \frac{F(z(a, b))}{z(a, b)} \cdot \phi^{\prime}\left(\frac{t}{z(a, b)}\right) .
$$

Then

$$
\begin{gathered}
\int_{0}^{a} \int_{0}^{b}\left\{\psi\left(\frac{\partial^{2}}{\partial s \partial t}(p)_{\alpha^{2}}(s, t) \cdot \varphi\left(\frac{\left|\frac{\partial^{2}}{\partial s \partial t}(u)_{\alpha^{2}}(s, t)\right|}{\frac{\partial^{2}}{\partial s \partial t}(p)_{\alpha^{2}}(s, t)}\right)\right) \cdot F^{\prime}\left(p(s, t) \cdot \varphi\left(\frac{|u(s, t)|}{p(s, t)}\right)\right)\right. \\
\left.+\psi\left(\frac{\partial^{2}}{\partial s \partial t}(z)_{\alpha^{2}}(s, t)\right) \cdot \frac{\partial}{\partial t}\left(F^{\prime}(z(s, t))\right)_{\alpha}(s, t) \cdot \frac{\frac{\partial}{\partial t}(z(s, t))_{\alpha}(s, t)}{\frac{\partial^{2}}{\partial s \partial t}(z)_{\alpha^{2}}(s, t)}\right\} d_{\alpha} s d_{\alpha} t \\
\leq \Phi\left(\int_{0}^{a} \int_{0}^{b} \frac{\partial^{2}}{\partial s \partial t}(p)_{\alpha^{2}}(s, t) \cdot \varphi\left(\frac{\left|\frac{\partial^{2}}{\partial s \partial t}(u)_{\alpha^{2}}(s, t)\right|}{\frac{\partial^{2}}{\partial s \partial t}(p)_{\alpha^{2}}(s, t)}\right) d_{\alpha} s d_{\alpha} t\right),
\end{gathered}
$$

where

$$
\psi(r)=r h\left(\phi\left(\frac{1}{r}\right)\right)
$$

and

$$
\Phi(r)=F(r) \cdot h\left(\frac{1}{r} \int_{0}^{a} \int_{0}^{b} \phi^{\prime}\left(\frac{t}{r}\right) d_{\alpha} s d_{\alpha} t\right) .
$$

Proof From (2.9), we have

$$
\varphi\left(\frac{|u(s, t)|}{p(s, t)}\right) \leq \frac{z(s, t)}{p(s, t)} .
$$

From (2.7), (2.15), (2.13) (2 times), Lemma 2.1, and noting that $h$ is a concave, increasing and using reverse Jensen's inequality, and (2.11) and (2.14), we obtain

$$
\begin{gathered}
\int_{0}^{a} \int_{0}^{b}\left\{\psi\left(\frac{\partial^{2}}{\partial s \partial t}(p)_{\alpha^{2}}(s, t) \cdot \varphi\left(\frac{\left|\frac{\partial^{2}}{\partial s \partial t}(u)_{\alpha^{2}}(s, t)\right|}{\frac{\partial^{2}}{\partial s \partial t}(p)_{\alpha^{2}}(s, t)}\right)\right) \cdot F^{\prime}\left(p(s, t) \cdot \varphi\left(\frac{|u(s, t)|}{p(s, t)}\right)\right)\right. \\
\left.+\psi\left(\frac{\partial^{2}}{\partial s \partial t}(z)_{\alpha^{2}}(s, t)\right) \cdot \frac{\partial}{\partial t}\left(F^{\prime}(z(s, t))\right)_{\alpha}(s, t) \cdot \frac{\frac{\partial}{\partial t}(z)_{\alpha}(s, t)}{\frac{\partial^{2}}{\partial s \partial t}(z)_{\alpha^{2}}(s, t)}\right\} d_{\alpha} s d_{\alpha} t \\
\leq \int_{0}^{a} \int_{0}^{b}\left\{\psi\left(\frac{\partial^{2}}{\partial s \partial t}(z)_{\alpha^{2}}(s, t)\right) \cdot F^{\prime}(z(s, t))\right. \\
\left.+h\left(\phi\left(\frac{1}{\frac{\partial^{2}}{\partial s \partial t}(z)_{\alpha^{2}}(s, t)}\right)\right) \frac{\partial}{\partial t}(z)_{\alpha}(s, t) \frac{\partial}{\partial t}\left(F^{\prime}(z(s, t))\right)_{\alpha}(s, t)\right\} d_{\alpha} s d_{\alpha} t
\end{gathered}
$$




$$
\begin{aligned}
= & \int_{0}^{a} \int_{0}^{b} h\left(\phi\left(\frac{1}{\frac{\partial^{2}}{\partial s \partial t}(z)_{\alpha^{2}}(s, t)}\right)\right) \cdot\left(\frac{\partial^{2}}{\partial s \partial t}(z)_{\alpha^{2}}(s, t) \cdot F^{\prime}(z(s, t))\right. \\
& \left.+\frac{\partial}{\partial t}(z)_{\alpha}(s, t) \cdot \frac{\partial}{\partial t}\left(F^{\prime}(z(s, t))\right)_{\alpha}(s, t)\right) d_{\alpha} s d_{\alpha} t \\
= & \frac{\int_{0}^{a} \int_{0}^{b} \frac{\partial^{2}}{\partial s \partial t}(F \circ z)_{\alpha^{2}}(s, t) \cdot h\left(\phi\left(\frac{1}{\frac{\partial^{2}}{\partial s \partial t}(z) \alpha^{2}(s, t)}\right)\right) d_{\alpha} s d_{\alpha} t}{\int_{0}^{a} \int_{0}^{b} \frac{\partial^{2}}{\partial s \partial t}(F \circ z)_{\alpha^{2}}(s, t) d_{\alpha} s d_{\alpha} t} \int_{0}^{a} \int_{0}^{b} \frac{\partial^{2}}{\partial s \partial t}(F \circ z)_{\alpha^{2}}(s, t) d_{\alpha} s d_{\alpha} t \\
\leq & h\left(\frac{\int_{0}^{a} \int_{0}^{b} \frac{\partial^{2}}{\partial s \partial t}(F \circ z)_{\alpha^{2}}(s, t) \cdot \phi\left(\frac{1}{\frac{\partial^{2}}{\partial s \partial t}(z) \alpha^{2}(s, t)}\right) d_{\alpha} s d_{\alpha} t}{\int_{0}^{a} \int_{0}^{b} \frac{\partial^{2}}{\partial s \partial t}(F \circ z)_{\alpha^{2}}(s, t) d_{\alpha} s d_{\alpha} t}\right) F(z(a, b)) \\
\leq & h\left(\frac{\int_{0}^{a} \int_{0}^{b} \frac{F(z(a, b))}{z(a, b)} \phi^{\prime}\left(\frac{t}{z(a, b)}\right) d_{\alpha} s d_{\alpha} t}{F(z(a, b))}\right) F(z(a, b)) \\
= & \Phi(z(a, b)) \\
= & \Phi\left(\int_{0}^{a} \int_{0}^{b} \frac{\partial^{2}}{\partial s \partial t}(p)_{\alpha^{2}}(s, t) \cdot \varphi\left(\frac{\left|\frac{\partial^{2}}{\partial s \partial t}(u)_{\alpha^{2}}(s, t)\right|}{\frac{\partial^{2}}{\partial s \partial t}(p)_{\alpha^{2}}(s, t)}\right) d_{\alpha} s d_{\alpha} t\right) .
\end{aligned}
$$

This completes the proof.

Remark 2.2 Putting $\varphi(x)=x$ in (2.12), we have

$$
\begin{aligned}
& \int_{0}^{b} \psi\left(\left|\frac{\partial^{2}}{\partial s \partial t}(u)_{\alpha^{2}}(s, t)\right|\right) \cdot F^{\prime}(|u(s, t)|) d_{\alpha} s d_{\alpha} t \\
& \quad \leq \Phi\left(\int_{0}^{a} \int_{0}^{b}\left|\frac{\partial^{2}}{\partial s \partial t}(u)_{\alpha^{2}}(s, t)\right| d_{\alpha} s d_{\alpha} t\right)-N_{\alpha}(a, b)
\end{aligned}
$$

where

$$
N_{\alpha}(a, b)=\int_{0}^{a} \int_{0}^{b} \psi\left(\frac{\partial^{2}}{\partial s \partial t}(z)_{\alpha^{2}}(s, t)\right) \cdot \frac{\partial}{\partial t}\left(F^{\prime}(z(s, t))\right)_{\alpha}(s, t) \cdot \frac{\frac{\partial}{\partial t}(z)_{\alpha}(s, t)}{\frac{\partial^{2}}{\partial s \partial t}(z)_{\alpha^{2}}(s, t)} d_{\alpha} s d_{\alpha} t .
$$

This inequality (2.16) is just a two-dimensional generalization of the following inequality which was established in [21]:

$$
\begin{aligned}
& \int_{0}^{b} \psi\left(D_{\alpha} p(t) \cdot \varphi\left(\frac{\left|D_{\alpha} u(t)\right|}{D_{\alpha} p(t)}\right)\right) \cdot F^{\prime}\left(p(t) \cdot \varphi\left(\frac{|u(t)|}{p(t)}\right)\right) d_{\alpha} t \\
& \quad \leq \Phi\left(\int_{0}^{b} D_{\alpha} p(t) \cdot \varphi\left(\frac{\left|D_{\alpha} u(t)\right|}{D_{\alpha} p(t)}\right) d_{\alpha} t\right),
\end{aligned}
$$

where $D_{\alpha} p(t)=D_{\alpha}(p)(t), \psi(r)=r h\left(\phi\left(\frac{1}{r}\right)\right)$ and $\Phi(r)=F(r) h\left(\phi\left(\frac{b}{r}\right)\right)$, and $h$ is a concave and increasing function on $[0, \infty)$.

\section{Acknowledgements}

The first author expresses his gratitude to professor G. Leng and W. Li for their valuable helps.

\section{Funding}

The first author's research is supported by the Natural Science Foundation of China $(11371334,10971205)$. The second author's research is partially supported by a HKU Seed Grant for Basic Research. 
Availability of data and materials

All data generated or analysed during this study are included in this published article.

\section{Competing interests}

The authors declare that they have no competing interests.

\section{Authors' contributions}

C-JZ and W-SC jointly contributed to the main results. All authors read and approved the final manuscript.

\section{Author details}

'Department of Mathematics, China Jiliang University, Hangzhou, P.R. China. ${ }^{2}$ Department of Mathematics, The University of Hong Kong, Hong Kong, P.R. China.

\section{Publisher's Note}

Springer Nature remains neutral with regard to jurisdictional claims in published maps and institutional affiliations.

Received: 21 December 2018 Accepted: 18 February 2019 Published online: 28 February 2019

\section{References}

1. Opial, Z:: Sur une inégalité. Ann. Pol. Math. 8, 29-32 (1960)

2. Agarwal, R.P., Pang, P.Y.H.: Opial Inequalities with Applications in Differential and Difference Equations. Kluwer Academic, Dordrecht (1995)

3. Agarwal, R.P., Lakshmikantham, V.: Uniqueness and Nonuniqueness Criteria for Ordinary Differential Equations. World Scientific, Singapore (1993)

4. Bainov, D., Simeonov, P.: Integral Inequalities and Applications. Kluwer Academic, Dordrecht (1992)

5. Li, J.D.: Opial-type integral inequalities involving several higher order derivatives. J. Math. Anal. Appl. 167, 98-100 (1992)

6. Mitrinovič, D.S., Pečarić, J.E., Fink, A.M.: Inequalities Involving Functions and Their Integrals and Derivatives. Kluwer Academic, Dordrecht (1991)

7. Cheung, W.S.: On Opial-type inequalities in two variables. Aequ. Math. 38, 236-244 (1989)

8. Cheung, W.S., Zhao, D.D., Pečarić, J.E.: Opial-type inequalities for differential operators. Nonlinear Anal., Theory Methods Appl. 66, 2028-2039 (2007)

9. Godunova, E.K., Levin, V.I.: On an inequality of Maroni. Mat. Zametki 2, 221-224 (1967)

10. Mitrinovič, D.S.: Analytic Inequalities. Springer, Berlin (1970)

11. Pachpatte, B.G.: On integral inequalities similar to Opial's inequality. Demonstr. Math. 22, $21-27$ (1989)

12. Pachpatte, B.G.: On Opial-type integral inequalities. J. Math. Anal. Appl. 120, 547-556 (1986)

13. Zhao, C.-J., Cheung, W.S.: Sharp integral inequalities involving high-order partial derivatives. J. Inequal. Appl. 2008, Article ID 571417 (2008)

14. Zhao, C.-J., Cheung, W.S.: On Opial-type integral inequalities and applications. Math. Inequal. Appl. 17(1), 223-232 (2014)

15. Pečarić, J.E.: An integral inequality. In: Srivastava, H.M., Rassias, T.M. (eds.) Analysis, Geometry, and Groups: A Riemann Legacy Volume, Part II, pp. 472-478. Hadronic Press, Palm Harbor (1993)

16. Pečarić, J.E., Brnetić, I.: Note on generalization of Godunova-Levin-Opial inequality. Demonstr. Math. 30, 545-549 (1997)

17. Pečarić, J.E., Brnetić, I.: Note on the generalization of Godunova-Levin-Opial inequality in several independent variables. J. Math. Anal. Appl. 215, 274-282 (1997)

18. Rozanova, G.I.: Integral inequalities with derivatives and with arbitrary convex functions. Mosk. Gos. Ped. Inst. Vcen. Zap. 460, 58-65 (1972)

19. Sarikaya, M.Z., Budak, H.: New inequalities of Opial type for conformable fractional integrals. Turk. J. Math. 41, 1164-1173 (2017)

20. Traple, J.: On a boundary value problem for systems of ordinary differential equations of second order. Zesz. Nauk. Uniw. Jagiell., Pr. Mat. 5, 159-168 (1971)

21. Sarikaya, M.Z., Bilisik, C.C.: Some Opial type inequalities for conformable fractional integrals. AIP Conf. Proc. 1991(1), 020013 (2018)

22. Sarikaya, M.Z., Budak, H.: Opial type inequalities for conformable fractional integrals. RGMIA Res. Rep. Collect. 19 Article ID 93 (2016) 